NASA Technical Memorandum 102266

\title{
Computation of Navier-Stokes Equations for Three-Dimensional Flow Separation
}

Ching-Mao Hung

(NASA-TM-102266) COMPUTATION OF NAVIER-STOKES EQUATIONS FOR

THREE-DIMFNSIONAL FLOW SEPARATION $22 \mathrm{P}$
(NASA)

CSCL OIA
N90-13353

Unclas

0252544

December 1989 


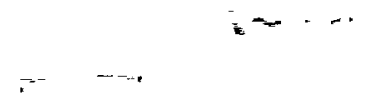




\section{Computation of Navier-Stokes Equations for Three-Dimensional Flow Separation}

Ching-Mao Hung

Ames Research Center, Moffett Field, California

National Aeronautics and

Space Administration

Ames Research Center

Moffett Field, California 94035 



\begin{abstract}
Computation of Navier-Stokes Equations for Three-Dimensional Flow Separation
\end{abstract}

\title{
Ching-mao Hung
}

NASA Ames Research Center, Moffett Field, CA

\section{SUMMARY}

Supersonic flows over a sharp and a flat-faced blunt fin mounted on a flat plate are simulated numerically. Several basic issues involved in the resultant three-dimensional stealy flow separation are studied. Using the same number of grid points, different grid spacings are employed to investigate the effects of grid resolution on the origin of the line of separation. Various shock strengths are used to study the so-called separated and unseparated boundary layer and to establish the existence or absence of secondary separation. The length of separation ahead of the flat-faced blunt fin, bifurcation of a horseshoe vortex, and the accessibility of a closed-type separation are investigated. The nsual interpretation of the flow field from previous studies and new interpretations arising from the present simulation are discussed.

\section{INTRODUCTION}

In the past, fluid clynamics has been divided into two branches, theoretical and experimental. Continuing advances in numerical methods and in computer capabilities have at some point qualified computation as a separate branch of fluid dynamics. Using the computer as a tool, computational fluid dynamics (CFD) is able to supplement the other two branches and to carry out its own research, development, and further advancement in fluid dynamics as a field of physical science.

Three-dimensional (3-D) flow separation is an excellent CFD problem. The problem has received considerable attention in recent years. In early studies, investigators have relied mainly on experimental observations of surface properties, such as static pressure and oil flow, or theoretical work which involves analysis of the governing equations of the kinematic properties of fluid flows, to deduce the basic features of the flow separation. Even though considerable progress has been made, the problem is so complicated that the two approaches have faced many difficulties and many more questions remain to be answered. With the advent of the supercomputer, CFD is now on the threshold of definitive explorations - solving the Navier-Stokes equations, and finding the details of the flow-field structure and the underlying physical processes.

Recently there have been a tremendous number of numerical solutions to the NavierStokes equations for complex geometries showing various 3 -D flow separations. Very often these studies were merely presenting their numerical capabilities and new applications. Instead of dealing with problems involving complicated geometries, the present paper will focus on supersonic flows over sharp or blunt fins mounted on a flat plate. This is one of the most common and important $3-D$ inviscid-viscous interaction problems and recently has attracted a substantial amount of interest (for example, refs. $1-7$ ). The primary purpose of this paper is to have an in-depth study of several basic issues regarding the $3-D$ flow separation.

To set the stage for discussion, some general concepts for the 3-D flow separation will be first briefly reviewed. In particular, the descriptions of bubble vs. free vortex 
layer and open vs. closed separations will be emphasized. The rest of the paper will then be devoted to discussion of the computed results of 3-D separation. The computed results can confirm, demonstrate or illustrate, but can not vigorously prove a physical concept. For each issue, we will present the usual interpretations from previous studies and discuss our new interpretations arising from the present simulations. We will present not only the results that agree with experimental observations, but more importantly, present results that suggest new conclusions and hopefully lead to a better understanding of fluid dynamics.

Only those 3-D viscous flows that are steady in the mean are considered here. The calculated separation to be discussed can be either laminar or turbulent and we shall make no effort to distinguish the basic separation pattern. (Whether the flow is compressible or not is also not distinguished.) Presumably we assume the general patterns are similar and the difference is a matter of degree, ralher than cliaracter. However, in reality turbulent separation has certain degrees of unsteadiness (e.g. see ref. 8), which may not be present for laminar flows. Future direct or large-eddy turbulence sinulations are needed for further understanding of unsteady turbulent $3-\mathrm{D}$ separation.

\section{CLASSIFICATIONS OF THREE-DIMENSIONAL SEPARATION}

For two-dimensional (2-D) steady flows, the criterion for the onset of separation is the vanishing of skin friction. This criterion is precise and convenient for practical applications. For 3-D steady flows, a similar criterion can not be used; the skin friction in general does not vanish, except at limited numbers of critical (or singular) points. This has inspired many researchers to establish a proper definition for flow separation in three dimensions. All the early work relied principally on (a) observations drawn from experimental studies utilizing flow visualization techniques and (b) mathematical patterns of streamlines immediately adjacent to the body surface (i.e., the limiting streamlines or skin-friction lines). It is a common observation that a necessary condition for 3-D flow separation is the convergence of oil-streak lines onto a particular line. The questions are (1) how and where this "separation" line starts and (2) what are the characteristics of this line.

Eichelbrenner (ref. 9) suggested that the line of separation is an envelope of limiting streamlines. (That means the liniting streamlines do intersect the line of separation.) The region behind the separation is inaccessible to the flow upstream. Maskell (ref. 10) elucidated the envelope criterion and, based on features of flow separation, went further to classify 3-D separation as either a bubble or a free-vortex layer (fig. 1). For the bubble type, there exists a surface of separation which isolates a quantity of fluid from the main flow. (The surface of separation either reattaches itself onto the solid surface or is closed downstream of the the body.) For the free-vortex-layer type, the separation streamlines are the skeleton of a vortex layer and the surface of separation rolls spirally into a discrete region of vorticity.

Note that, while the bubble separation is well defined, the free-vortex-layer separation is not. Maskell has never described how and where the free vortex layer originates by itself. He suggested that combinations of the bubble and the free-vortex-layer separation are the most likely flow patterns. Whenever a bubble forms between two distinct lines of separation, a free vortex layer originates from the bubble surface and a mixed flow results. However, this leads to the controversy of merging two separation surfaces to become a free vortex layer. It corresponds to the controversy of intersection of two streamlines at a regular point in 2-D flow field. Furthermore, while the bubble separation is well defined, the reattachment of a separation surface itself onto the solid surface, as required for form- 
ing a bubble, is structurally unstable. Therefore, the existence of the completely closed separation bubble becomes questionable (see discussion later).

Instead of dealing with the flow filed feature of separation away from the wall, Wang (ref. 11) concentrated on the pattern of limiting streanlines near the body surface. He also was in favor of the envelope concept. Based on the accessibility from flow upstream, he classified the separation as closed (inaccessible) or open (accessible). (The concept of bubble and inaccessibility will be critically discussed later.)

Lighthill (ref. 12) and Legendre (ref. 13) pioneered the theoretical approach and assumed that the pattern of limiting streamlines (or skin-friction lines) be considered as trajectories having properties consistent with those of a continuous vector field. There is one and only one principal trajectory passing through any regular point. Thus, the physics of the vector field is governed by a set of autonomous ordinary differential equations. Also the flow pattern can be characterized by the type and number of singular points and rules governing the relations between them. The line of separation is defined as a skin-friction line which issues from both side of a saddle point of separation and, after embracing the body, disappears into a nodal point of separation. Note that the "separation" is implicitly clefined and described by the flow characteristics at the saddle and nodal points of separation and along the skin-friction line connected between them. In contrast to the envelope concept, flow separation in this view has been considered as the convergence (but not intersection) of skin-friction lines onto the line of separation. The advantage of this approach is that mathematical theory, such as topological structure, structural stability, bifurcation theory, and qualitative theory of differential equations, can be employed. Lighthill and Legendre still considered a $3-D$ separation inaccessible to the upstream fluid. Legendre (ref. 13) insisted that 3-D separation exists only if at least one wall streamline is prolonged within the fluid by a whole surface forming a barrier to the upstream flow, either in completely preventing it from penetrating a downstream domain, or in obliging it to pass around the barrier.

Tobak and Peake (ref. 14) also adopt the concept of convergence of limiting streamline. However, they proposed a distinction between local and global separations. For the global separation, as described by Lighthill and Legendre, the line of separation originates from a saddle point and its critical points are in accord with the topological theory, while for the local separation, the line of separation starts from a noncritical point.

As one can see, the so-called global and local separations are very similar, aside from some conceptual differences, to Wang's closed and open separations, respectively. In the present paper, we will follow the concept of convergence of limiting streamline and will be in favor of Tobak and Peake's more precise global-and-local definition, but adopt Wang's simple closed-and-open terminology. Therefore, we define (fig. 2) an open-type separation as that for which the line of separation starts from a non-critical point, while for a closed-type separation the line of separation originates from a saddle point. Note 1.hat whether an open separation can start from a regular point is still a matter of current debate. The exact definition and location of the onset of an open separation remain unclear and controversial. The origin of its associated vortex layer and its corresponding vortex core also remain unanswered. It is beyond the scope of this paper to debate these issues.

In most cases, the patterns of the skin-friction line provide the liniting flow behavior near the body surface, and they enable us to extract certain information about the whole flow field. However, as pointed out by Dallmann (ref. 15), surface streamline patterns alone cannot provide a unique interpretation of the flow field. The study of 3-D flow separation requires the examination of the surface skin-friction lines as well as the external flow field itself. Numerical computation with graphic techniques provides this requirement and allows one to visualize the entire flow field in a dynamic sense. 


\section{SUPERSONIC FLOW OVER A SHARP FIN}

The first case to be discussed is a simulation of flow through a shock wave, generated by a sharp fin, glancing across a laminar boundary layer growing over a flat plate (fig. 3 ). The governing equations of the analysis are the time-dependent, compressible NavierStokes equations incorporating the concept of the thin-layer approximation (ref. 16) in all three directions. The flow is assumed laminar and the wall is adiabatic. A numerical procedure developed by Hung and Kordulla (ref. 17) is used. The basic numerical scheme is MacCormack's (ref. 18) explicit-implicit predictor-corrector algorithm. The solution is carried out until it converges to a steady state. Details of the numerical technique and boundary conditions are discussed in reference 17 .

Several basic issues concerning the resultant 3-D flow separation will be studied. Using the same number of grid points, different gricl spacings are employed to investigate the effects of grid resolution on the origin of the line of separation. Various shock strengths (generated by use of different fin angles) are used to study the so-called separated and unseparated boundary layer and to establish the existence or absence of the secondary separation.

Figure 4 shows a typical mesh system of $57 \times 45 \times 27$ points for a sharp fin on a flat plate. The apex of the fin (at $\mathrm{x}=0.0$ ) is placed at a distance $L=9 \mathrm{~cm}$ from the flat plate leading edge and this distance $L$ is used as the characteristic length in the present study. Here $(x, y, z)$ and $(I, J, K)$ are used in the conventional sense of streamwise, crossflow, and vertical directions. Using the same number of grid points, three different grid spacings are employed. The coarse grid is uniform in the streamwise direction and geometrically stretched from the fin and plate ( in the J-and $\mathrm{K}$-directions). The medium grid has additicnal geometric stretching in the streamwise direction from the apex of the fin. The fine grid has finer spacing ( compared to the medium grid) near the fin in the Jdirection and near the apex of the fin in the streamwise direction. To avoid over-stretching in the outer region, several zones with different stretcling factors are used in the I- and J-directions. Smooth transition in grid spacing is ensured from one zone to another. The grid spacing parameters are listed in table 1.

Figure 5 shows a comparison of surface pressures at $y=5 \mathrm{~cm}$ for a wedge angle $\theta=6^{\circ}$. All results are in good agreement, except that the computed result of Degrez (ref. 19) shows a "dip" after the separation. The present results and the experimental data do not show the appearance of the dip in surface pressure. The fine grid result (not shown in fig. 5) is very close to the medium grid result. This indicates that our grid refinement does not affect the prediction of surface pressure.

\section{Origin of the Line of Separation}

Reference 6 contains an extensive study of the flow-field structure of this geometry for a turbulent boundary layer. It is commonly accepted that the primary separation is a consequence of the high pressure, recovered from the shock system, which induces flow from the fin surface and forces the boundary layer off the sidewall. The question is, where is the origin of the line of primary separation? Figure 6 shows particle traces of the result based on a two-equation model as described in reference 6 for the first mesh points above the sidewall $(\mathrm{K}=2)$. This particle trace is constructed by a time integration of velocity components restricted to the plane of $K=2$. Since the plane of $K=2$ is very close to the flat plate, (normally it would have a resolution smaller than the size of an oil particle), the particle integrations are treated as surface particle traces and are considered equivalently as a simulation of oil flow in the experiment and as a simulation of skin-friction lines in the theoretical approach. The particle traces in figure 6 indicate that the line of separation 
originates somewhere in the plate away from the apex of the fin and that this feature is an open-type separation.

Figures $7(\mathbf{a}-\mathbf{c})$ show surface particle traces for the sequence of three grid refinements. For the coarse grid, the separation is an open type. As the grid spacing near the leading edge is refined, the starting point of the open-type separation moves and eventually the separation becomes a closed type. This clearly demonstrates that the choice of grid resolution can affect the "calculated" topology. 'The coarse grid simply cannot resolve the vortex structure, while the fine grid can. As the wedge angle increases to $12^{\circ}$, the vortex structure is large enough that the medium grid ( not shown here) is able to reveal a closed-type separation.

While the existence or not of an open-type separation is still in question, we believe that some of the numerically observed open-type separations result from insufficient grid resolution (see sketch, fig. 8). Similarly, experiments also have resolution problems, such as the size of oil droplets. Some of the experimentally observed open-type separations may be the result of low resolution of the device and facility.

Hereafter the fine-grid result will be used for discussion, except for cases specially mentioned.

\section{Secondary Separation}

The second question is the existence of a secondary separation. Experiments (for instance refs. 20 and 21 ) very often show the appearance of another oil-accumulation line behind the line of primary separation. This has been interpreted as indicating the existence of a secondary separation, (fig. 9). The plots of velocity at the first mesh point above the flat plate, $\mathrm{K}=2$, (fig. $10(\mathrm{a})$ ) also show that, in addition to the outermost primary separation line, (not clearly visible on the figure), there is a second "line" of clustering or coalescence of velocity vectors. However, based on the result of surface particle traces (fig. 10(b)) this is not a line of separation - it is merely a demarcation between regions of high and low surface skin friction. (The lengths of the velocity vectors are proportional to the magnitude of the skin friction.) One might imagine that, in a transient stage of an experiment, comparatively more "oil" can be driven in by the high skin friction from the right and less oil carried out to the left near the region of strong variation in skin friction. (For convenience of discussion, here left or right refers to the orientation of one facing the streamwise direction.) Hence the surface may show a temporary accumulation of oil around this second line. Even as the wedge angle increases up to $12^{\circ}$ (fig. 11(a)), our calculations still show no evidence of the existence of secondary separation. Indeed for the $12^{\circ}$ case, behind the obvious primary separation line, there is a region that the particle traces show strong convergence from one side and slow divergence from the other side. ( These traces eventually converge to the primary separation line. ) Hence a high clustering of particle traces in that region occurs. From the plot of velocity vectors in the plane $\mathrm{K}$ $=2$ (fig. 11(b)), one can see that this region is associated with the drastic change of the surface skin friction in both magnitude and direction. The flow features near the wall are strongly affected by the surface pressure. Their relations and connections can be seen from figure 11(c). A strong surface pressure gradient induces a high skin friction and a high velocity near the sidewall. This results in a divergence of particle traces (fig. 11(b)). After the strong pressure gradient, there is a region of drastic change of skin friction in both magnitude and direction and hence a resulting appearance of clustering of velocity vectors near the surface. The strong pressure gradient appears mainly to the right of the inviscid shock location (fig. 11(c)) and the clustering of velocity appears on the left (fig. 11(b)). Note that the appearance of the clustering of particle traces (fig. 11(a)) does not coincide with the clustering of the velocity vectors near the wall. Instead, it is close to the pressure 
plateau region ( shown as a band with the clustering of the velocity vectors on its right). It is this pressure plateau region that causes the particle traces to run almost parallel to each other before they finally converge to the primary separation line. We suspect that variations in surface-flow-visualization techniques could also result in different locations of temporary clustering of surface-streak lines.

Note that Degrez's calculation showed a noticeable "dip" of pressure for the $6^{0}$ case (see fig. 5). When it is strong enough, this drop in pressure can significantly retard the primary separated flow (passing beneath the shock system in the opposite y-direction to the main flow ) and lead to a secondary separation (see fig. 12 of ref. 19). In the present calculation, the pressure shows a plateau region and there is no secondary separation for either the $6^{\circ}$ or $12^{\circ}$ case. (The $12^{\circ}$ case has a large plateau region with a slight dip of pressure.) As discussed above, a drastic change of velocity leads to a substantial change of skin friction which might also result in a temporary accumulation of oil flow on the surface. Therefore, it is possible that an accumulation of oil flow on the surface in an experiment is not necessarily a line of separation, contrary to the usual inference.

It should be noted that we don't know whether or not the appearance of secondary oil-accumulation in an experiment is caused by a secondary separation. One possible alternative is suggested here. Furthermore, there might be other mechanisms in an experiment, especially for the turbulent case, that could lead to an oil accumulation on the surface. Further detailed studies are needed to answer these questions.

\section{Absence of Separation}

Whether the boundary layer on the plate is separated or not is usually (for instance, ref. 22) determined by comparing the turning angle of the liniting streamline with the glancing shock angle in the interaction region (fig. 12). As shown in both figures 10(b) and $11(\mathrm{a})$, there is clearly a line, with clustering of particle traces, that originates from a saddle point with turning angle greater than the angle of the glancing shock, and both flows are considered separated. As the wedge angle decreases, one would expect that the turning angle of the skin friction line will decrease and eventually become smaller than the angle of the glancing sliock. The flow then will be classified as attached as stated above.

In the present paper a case with $2^{\circ}$ wedge angle was calculated with the fine grid distribution. Figure 13(a) shows traces of particles for which the origins are almost the same as those of the $6^{\circ}$ case (fig. 7). In contrast to figures 7 and $11(\mathrm{a})$, there is no obvious line of convergence of particle traces and the turning of skin-friction lines is smaller than the glancing shock angle; this would conventionally be interpreted as an attached case. However, a close examination of the particle traces near the apex (fig. 13(b)) shows that actually the flow is separated. Even though it is very small, the separation is also a closed type, and the structure is topologically the same as that for the previous $6^{\circ}$ and $12^{\circ}$ cases. Actually, all three cases are topologically the same as the structure of a blunt-fin flow field (see later on discussion).

One may imagine that, under certain conditions, this type of flow may not separate. However, the conventional method of interpretation using the turning angle of the boundary layer compared to the glancing shock angle as a criterion for separation is not uniformly valid, as demonstrated above.

Another point should be mentioned here. Figures 12 and 21 of reference 7 sketched that, at a low wedge angle, the boundary layer on the sidewall was separated from a saddle point as a closed type of separation on the line of symmetry near the fin apex, and then gradually became attached away from the fin. (This was claimed in reference 7 as an open-type separation.) The question arises as to where and how the separation ends. The 
author believes the simplest explanation is that for this case, the definition of separation based on turning angle and oil accumulation in experimental observation fails. Because of boundary layer growth on the fin, the shock wave is stronger and hence the pressure rise is higher near the leading edge of the fin than at a position downstream. The difference in pressure rise changes the turning angle and degree of oil accumulation. Based on the concept of continuity, limiting streamlines would not join together except at a singular point. A line of separation, once it originates from a saddle point, will either continue going downstream to infinity or terminate at a singular point. In other words, once it is separated, the flow can not gradually be reattached without a singular point, according to topological imperatives.

\section{SUPERSONIC FLOW OVER A FLAT-FACED BLUNT FIN}

The second problem to be presented is supersonic flow over a flat-faced blunt fin mounted on a flat plate (fig. 14). The bow shock causes the boundary layer to separate from the plate ahead of the blunt fin resulting in a separated flow region composed of horseshoe vortices near the surface and a lambda-type shock pattern ahead of the fin. The shock wave emanating from the separated flow region (separation shock) interferes with the bow shock, and causes intense heating and high pressure locally around the leading edge of the protuberance.

The flows considered are turbulent. The governing equations are the time-dependent, compressible Reynolds-averaged Navier-Stokes equations. An algebraic eddy-viscosity turbulence model by Baldwin and Lomax (ref. 16), with a "modified distance" (ref. 5), is used to close the system of equations. The numerical procedure developed by Hung and Kordulla (ref. 17) was also used. The question of length of separation, structure of horseshoe vortex and the accessibility of the closed-type separation will be discussed.

\section{Length of Separation}

For a hemicylindrical blunt fin, it has been observed from experiments (e.g., refs. 1 and 4 ) and numerical simulations (ref. 5) that many of the important flow length scales are primarily dependent on the diameter of the fin leading edge, $D$, and only weakly dependent. on the free-stream Mach number, $M_{\infty}$, and thickness, $\delta$, of the incoming boundary layer. Variation of the thickness of incoming boundary layer by a factor of 10 in reference 5 produced approximately the same size of horseshoe vortex and therefore the same spatial extent of the interaction. The length of separation along the line of symmetry is about 2.0 to $2.5 \mathrm{D}$, provided the inconuing boundary layer is turbulent. As the bluntness of the leading edge increases, one can expect the length of separation to increase. The question is how much this length will increase.

To study the effect of bluntness on the length of separation, let's consider the extreme case, a flat-face fin. As sketched in figure 15, the length of separation is about 2.5 D on the line of symmetry for a hemicylindrical blunt fin. As the hemicylindrical portion is removed, the bow shock will follow as sketched. Intuitively, one would expect that the location of separation should stay roughly the same. That means the length of separation would increase to about $3.0 \mathrm{D}$, or slightly less. Surprisingly, it increases even more (by a factor about two for the flat-faced fin). The length of separation is about $5.2 \mathrm{D}$ for the present computation and about $5.5 \mathrm{D}$ experimentally (ref. 23). This can be seen clearly in the plots of simulated "oil flow" on the flat plate (fig. 16). The calculated results confirm the observation from experiments (refs. 23 and 24) of the drastic increase in separation length for flow over a flat-faced fin. Empirically there may be several parameters, such as drag coefficient, and bluntness, to correlate, but basically we still don't understand the 
reason for this drastic increase.

\section{The Existence of Secondary Separation}

Figure 17 shows the comparison of surface pressure along the symmetry line for the flat-faced blunt fin. (The plot of the $u$-velocity at the grid points of $K=2$ is also shown for later discussion.) The agreement between the computed result and the experiment data (ref. 23) is reasonably good. The measured pressure shows the appearance of a small second pressure peak (or a "kink"), which, in reference 23, was interpreted as an indication of the existence of a secondary separation and the development of an additional pair of vortices. Another question arises with the appearance of a secondary separation. Whenever there is a secondary separation, the issue is whether the main horseshoe vortex will remain as one or bifurcate into two vortices. Sedney and Kitchens (ref. 25) suggest that the main horseshoe vortex will bifurcate and that there will be an even number of vortices. As shown in figure 18 , the process starts with two vortices - one main horseshoe vortex and one small one near the corner. As secondary separation appears, the main horseshoe vortex bifurcates, and the vortices develop by pairs, in even numbers. For certain flow conditions, the existence of as many as six vortices has been inferred from an experimental oil flow (ref. 25).

Figure 19 shows calculated particle paths in the plane of symmetry. The horseshoe vortex does indeed bifurcate into two vortices of the same sign. However, there is no secondary separation under the main horseshoe vortices, and there is an odd number of vortices - three in this case. There are two peaks in the reverse u-velocity near the wall $(\mathrm{K}=2)$ under the core of these two vortices (see fig. 17). The reverse velocity near the wall between these two peaks slows down and then speeds up. That leads to the appearance of a kink in the surface pressure. The present calculation still has, in both the $\mathrm{x}$ - and $\mathrm{y}$-directions, very good grid-point resolution in the region around the pressure kink. Therefore, we don't think that grid-point resolution is an issue in the present computation concerning the non-existence of a secondary separation.

Under different flow conditions, this deceleration of the reverse velocity eventually may lead to a secondary separation. This means that for this type of flow the appearance of a kink in pressure is only a necessary condition for the existence of secondary separation, not a sufficient condition. In the other words, even though the pressure field and secondary separation might interact with each other, the secondary separation is generally controlled by the pressure field, rather than vice versa.

\section{Structures of Horseshoe Vortices}

It has been generally believed that the structure of horseshoe vortices is in accord with the jet-maze model of Norman (ref. 26), as sketched in figure 18 and re-sketched in figure 20(a) for the case of no secondary separation. The separation point (fig. 20(a)) is connected to the outer vortex (indicated as 2 ). The fluid between a and $b,[a-b]$, and between $c$ and $d,[c-d]$ (indicated as 2 ) is entrained in the outer vortex. The fluid between [b-c] (indicated as 1) in the inner vortex, and between [d-e] (indicated as 3) in the third vortex near the corner. The fluid above $[\mathrm{e}]$ remains outside the vortices. The structures based on the present calculations (fig. 19) are re-sketched for clarity in figure $20(\mathrm{~b})$. The separation point, $S$, ties with the inner vortex (indicated as 1 ) and the four "layers" of fluid entrained in the three vortices are now marked $1-2-1-3$, instead of $2-1-2-3$ as in figure 20(a). (These features of entrainment are discussed in the next Section, for the accessibility of a closed-type separation.)

Note that, in figures 18 and $20(\mathrm{~b})$, topologically the attachment point corresponding 
to the primary separation point $S$ is point $A$ on the fin, and is not, as conventionally referred, point $A 1$ on the plate. The attachment point $A 1$ corresponds to the small separation $S 1$ near the corner. If the flow is assumed incompressible, there are lines of zero vorticity joining the separation and attachment points $S-A$ and $S 1-A 1$, respectively. It can also be argued that, for an incompressible flow, there are corresponding pressure minima ahead of the separations $S$ and $S 1$, and pressure maxima behind the reattaclments $A$ and $A 1$.

\section{Accessibility and Openness}

Based on the above observations for computed results, we can delineate the differences between $2-D$ and $3-D$ separations. In two dimensions, the separation line either connects to a reattachment point and forms a recirculation bubble, or extends to infinity and forms as a wake. In either case, there is a dividing streamline such that the region behind the separation is not a part of the main flow and is inaccessible to the upstream fluid particle. In three dimensions this is not true.

In figure 16, the critical point $S$ is a saddle point and there is a skin-friction line $S-P$, on the plate, which emanates from point $S$. This type of separation is classified as a closed-type separation, as described in the classification of $3-D$ separation. Under the influence of the conception of $2-D$ separation, it has been commonly stated that the separation region behind the separation line $S-P$ is inaccessible to the flow coming from upstream (see for instance, refs. 11 and 13). However, this inaccessibility is only valid in the limit of fluid particles moving near/on body surface only. As has been demonstrated above, the separation region of this type is accessible to fluid upstream. The skin friction lines ahead of $S-P$ (fig. 16) asymptotically approach but can not get into the region behind the separation line $S-P$. However, based on the concept of limiting streanline, a spiral vortex sheet is generated by an infinite number of streamlines (including the separation line $S-P$ on the surface ) emanating from the saddle point $S$. Because of the spiral nature of the vortex separation, the fluid above the body surface can access the separation region behind $S-P$. In the present case, there are four layers of fluid entraining into three vortices in a manner as shown in figure $20(\mathrm{~b})$.

Note that the fluid particles separated from saddle point $S$ do not "reattach" to the surface. It is the fluid particles along the "stream tubes" (d) and (e), in figure 20(b), that attach to the nodal points $A 1$ and $A$ on body and "wet" the plate surface belind the line of separation $S-P$ and the fin surface. Since the fluid particles reaching the attachment points $A 1$ and $A$ are not the fluid particles that have previously separated; therefore, in 3-D flow, it is an "attachment" point, not a "reattachment" point as used in 2-D flow. On the body surface, the separation points $S$ and $S 1$ are saddle points and the attachment points $A$ and $A 1$ are nodal points; the connections from $A 1$ to $S$ and $A$ to $S 1$ are stable structures. In the plane of symmetry, the separation points $S$ and $S 1$ are half-saddle points, and the attachment points $A$ and $A 1$ are also half-saddle points. Mathematically, a connection of saddle point to saddle point in a continuous vector field is structurally unstable. (The 2-D separation and reattachment is structurally unstable, and is a transient state of a $3-\mathrm{D}$ separation.) Hence the separation point $S$ cannot be connected to the attachment point $A$ by a single structurally stable streamline. (It is similarly true for $S 1$ and $A 1$.) Theoretically, there is no steady $3-\mathrm{D}$ separation which is totally closed by a separation surface. (A perfectly axisymmetric separation is considered as a 2-D separation.) There must be some fluid flowing in and some fluid flowing out. All steady 3-D separation surfaces are a kind of vortex sheet in structure. In the words, all steady 3-D separations are a free-vortex-layer type; there is no bubble-type 3-D separation as conceived by Maskell (ref. 10). 
Finally, we would like to point out that in a 3-D separation, except for some simple flow structures, an attachment process is not a reverse of a separation process, or vice versa. As discussed above (figs. 16, 19 and $20(b)$ ), the flow separation originates from the saddle point $S$ and has a separation surface (free vortex layer) that spirals into a vortex or vortices. The constant feeding of vorticity from the free vortex layer into the vortex core makes the spiral-inward vortex structure stable. The attachment is associated with the nodal point $A$ and there is no "attachment" surface. The characteristics of saddle and nodal points are completely different. A reversed "separation process" would imply the existence of a stable, spiral-outward vortex or vortices which so far has never been observed in a steady flow. Similarly, a reversed "attachment process" would not produce the features as described for a separation. This is quite different from the 2-D separation phenomena.

\section{CONCLUSION AND REMARKS}

Computations of supersonic flow over a sharp and blunt fin mounted on a plate were used to study 3-D steady flow separation. For the sharp fin case, separation of the boundary layer on the flat plate was investigated for various grid refinements and fin wedge angles. The calculated results have demonstrated that grid resolution can affect the "calculated" topology. For the coarse grid, the separation is an open type. As the grid spacing near the leading edge is refined, the starting point of the open-type separation moves upstream and eventually the separation becomes a closed type. In the opinion of the author, some of the numerically observed open-type separations (for example, fig. 4) results from insufficient grid resolution. Similarly, experiments have resolution problems, and some of the experimentally observed open-type separations may well be due to low resolution of the flow visualization techniques.

Based on the computation of surface particle traces, no secondary separation was found in the present study. A secondary oil-accumulation line has been conjectured to be a demarcation between regions of high and low surface skin friction.

In a calculation with a $2^{\circ}$ sharp-fin angle, there is no obvious line of convergent particle traces and the turning angles of skin-friction lines are smaller than the glancing shock angle. This is conventionally interpreted as an attached flow. However, a close examination of the particle traces near the apex has shown that actually the flow is separated, and the structure is the same topologically as that for the blunt-fin flow field.

For the flat-faced blunt fin case, the following observations and conclusions are made.

1. The length of separation increases to about $5.2 \mathrm{D}$, compared with about 2.0 to 2.5 $D$ for the typical hemi-cylindrical results, and the present numerical results confirm these experimental observations.

2. Even though there is a "kink" in the pressure distribution for the present case, the main horseshoe vortex bifurcates into two and there is no secondary separation under the main horseshoe vortices. In this case three vortices are present; therefore one can conclude that the number of vortices is not always an even number.

3. For the case investigated, the separation point is connected to the inner (downstream) horseshoe vortex, rather than the outer (upstream) one. The four layers of fluid marked $1-2-1-3$ entrain into three vortices as shown in figure 20(b).

4. The concept of a closed 3-D separated region being inaccessible is valid only in the linit of particles moving near the body surface. The flow particles above the surface are able to access the separated region through the attachment node and spiral nalure of the separation. Indeed, there is no 3-D separation which is totally closed by a separation 
surface; there must be some fluid flowing in and some fluid flowing out. All 3-D separation surfaces are a kind of vortex sheet. In general, for 3-D separation, an attachment is not the reverse of a separation, or vice versa.

\section{REFERENCES}

1. Kaufman, L.G.; Korkegi, R.H.; and Morton, L.C.: Shock Impingement Caused by Boundary Layer Separation Ahead of Blunt Fin. ARL 72-0118, Wright-Patterson Air Force Base, Ohio, 1972.

2. Hung, C. M.; and MacCormack, R. W.: Numerical Solution of Three-Dimensional Shock-Wave and Turbulent Boundary-Layer Interaction. AIAA J., vol. 16, no. 12, Dec. 1978, pp. 1090-1096.

3. Horstman, C. C.; and Hung, C. M.: Computation of 3-D Turbulent Separated Flow at Supersonic Speed. AIA A Paper No. 79-0002, 1979.

4. Dolling, D.S.; and Bogdonoff; S.M: Blunt Fin-Induced Shock Wave/ Turbulent Boundary Layer Interaction. AIAA J., vol. 20, no. 12, 1982, pp. 1674-1680.

5. Hung, C. M.; and Buning, P. G.: Simulation of Blunt-Fin-Induced Shock Wave and Turbulent Boundary-Layer Interaction. J. Fluid Mech., vol. 154, May 1985, pp.163-185

6. Knight, D. D.; Horstman, C. C.; Shapey, B.; and Bogdonoff, S.: The Flowfield Structure of the 3-D Shock Wave-Boundary Layer Interaction Generated by a $20^{\circ}$ Sharp fin at Mach 3. AIAA Paper No. 86-0343, 1986.

7. Fomison, N. R.; and Stollery, J. L.: The Effects of Sweep and Bluntness on a Glancing Shock Wave Turbulent Boundary Layer Interaction. AGARD CP 428, Paper 8, 1987.

8. Dolling, D.S.: Unsteadiness of Supersonic and Hypersonic Shock-Induced Turbulent Boundary Layer Separation. AGARD-FDP/VKI Special Course on "ThreeDimensional Supersonic and Hypersonic Flows Including Separation", May 8-12, 1989.

9. Eichelbrenner, E. A.: Three-Dimensional Boundary Layers. Annu. Review of Fluid Mech. vol. 5, 1973, pp. 339-360.

10. Maskell, E. C.: Flow Separation in Three Dimensions. RAE report No. Aero. 2565, Nov. 1955.

11 Wang, K.C.: Boundary Tayer Separation in Three Dimensions. Reviews in Viscous Flow, Proc of the Lockheed - Georgia Company Viscous Flow Symposium, June 1976 , pp. 341-414.

12. Iighthill, M.J.: Attachment and Separation in Three-Dimensional Flow. In Laminar Boundary Layers edited by L. Rosenhead, Oxford Univ. Press, 1963, pp.72-82.

13. Legendre, R.: Regular or Catastrophic Evolution of Steady Flows Depending on Parameters. Rech. Aerosp. vol. 1982-4, pp. 41-49, 1982.

14. Tobak, M.; and Peake, D. J.: Topology of Three-Dimensional Separated Flows. Annu. Review of Fluid Mech. vol. 14, 1982, pp. 61-85.

15. Dallmann, U.: Topological Structure of Three-Dimensional Flow Separations. DFVL R-IB 221-82 A 07, 1982.

16. Baldwin, B. S.; and Lomax, H.: Thin-Layer Approximation and Algebraic Model for Separated Turbulent Flows. AIAA Paper No. 78-257, Jan. 1978. 
17. Hung, C. M.; and Kordulla, W.: A Time-Split Finite-Volume Algorithm for ThreeDimensional Flowfield Simulation. AIAA J., vol. 22, no. 11, 1984, pp. 1564-1572.

18. MacCormack, R.W.: A Numerical Method for Solving the Equations of Viscous Flow. AIAA J., vol. 20, no. 9, 1982, pp. 1275-1281.

19. Degrez, G.: Computation of a Three-Dimensional Skewed Shock Wave Laminar Boundary Layer Interaction. AIA A Paper No. 85-1565, 1985.

20. Aso, S.; Hayashi, M.; and Tan, A. Z.: The Structure of Aerodynamic Heating in Three-Dimensional Shock Wave/Turbulent Boundary Layer Induced by Sharp and Blunt Fins. AIAA paper No. 89-1854, June 1989.

21. Brosh, A.; Kussoy, M.I.; and Hung, C.M.: An Experimental and Numerical Investigation of the Impingement of an Oblique Shock Wave on a Body of Revolution. AIAA J., vol. 23 , no. 4 , June 1985 , pp. 840-846.

22. Green, J.E.: Interactions Between Shock Wave and Turbulent Boundary Layers. Progress in Aerospace Sciences, vol. 11, Pergamon Press at Oxford, 1970, pp. 235340.

23. Rodi, P. E.; and Dolling, D. S.: Experimental Study of the Effects of Leading Edge Geometry on Fin Induced Turbulent Separated Flow at Mach 5: Preliminary Results. AIAA Paper No. 86-0344, Jan. 1986.

24. Saida, N.: Separation Ahead of Blunt Fins in Supersonic Turbulent Boundary Layers. Presented at IU'TAM Symposium on Turbulent Shear Layer/Shock Wave Interaction," Palaiseau, France, Sept. 1985.

25. Sedney, R.; and Kitchens, C. W.,Jr.: The Structure of Three-Dimensional Flows in Obstacle-Boundary Layer Interaction. Flow Separation, AGARD CP-168, Paper 37, 1975 .

26. Norman, R.S.: On Obstacle Generated Secondary Flows in Laminar Boundary Layers and Transition to Turbulence. Ph.D. Thesis, Illinois Inst. Technol., 1972.

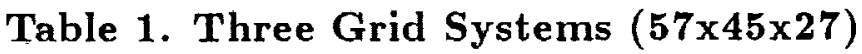

\begin{tabular}{|c|c|c|c|}
\hline & coarse & medium & fine \\
\hline$\triangle x \min$ & 0.10 & 0.02 & .01 \\
\hline$\triangle y \min$ & 0.0025 & 0.0025 & 0.0015 \\
\hline$\triangle z \min$ & 0.001 & 0.001 & 0.001 \\
\hline plate leading edge & $\mathrm{I}=4$ & $\mathrm{I}=3$ & $\mathrm{I}=3$ \\
\hline fin leading edge & $\mathrm{I}=14$ & $\mathrm{I}=22$ & $\mathrm{I}=22$ \\
\hline
\end{tabular}




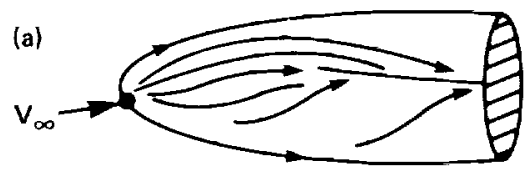

SURFACE

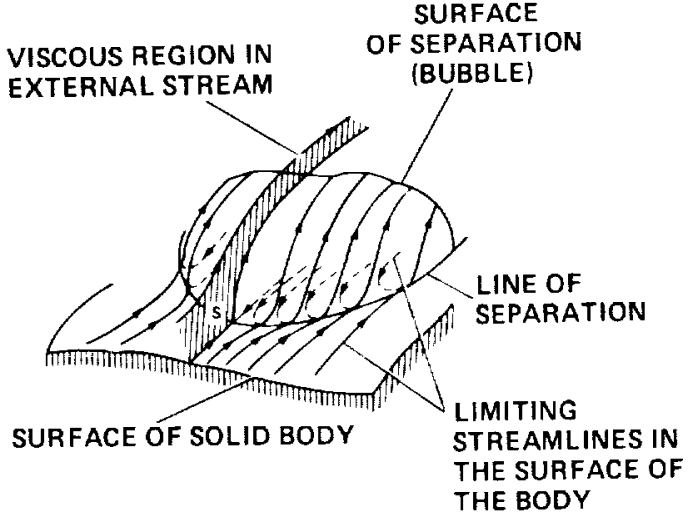

(a) BUBBLE

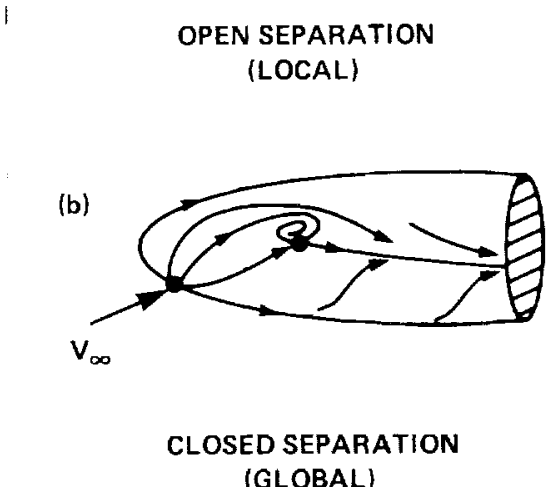

Figure 2.- Open vs. closed separation.

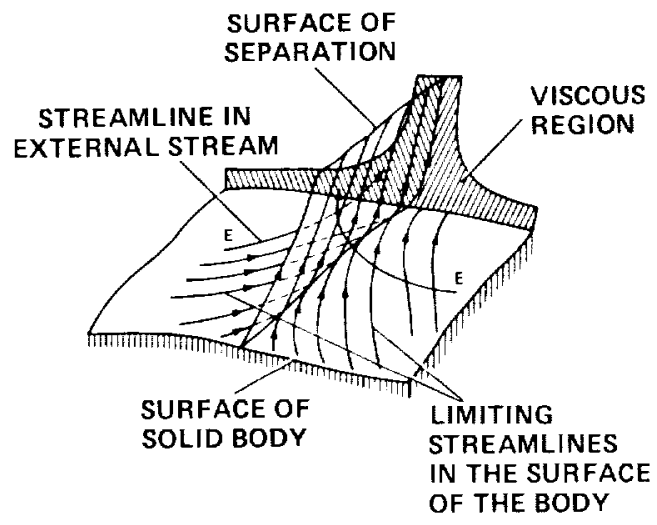

(b) FREE VORTEX LAYER

Figure 1.- Classification of three-dimensional separation by Maskell (ref. 10).

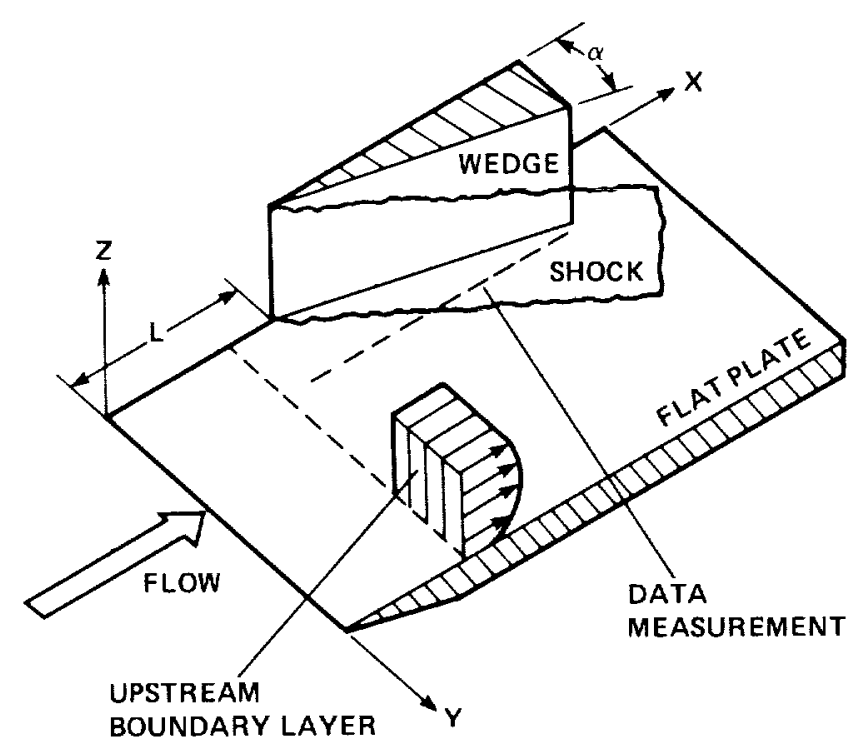

Figure 3.- Supersonic flows over a sharp fin on a plate. 


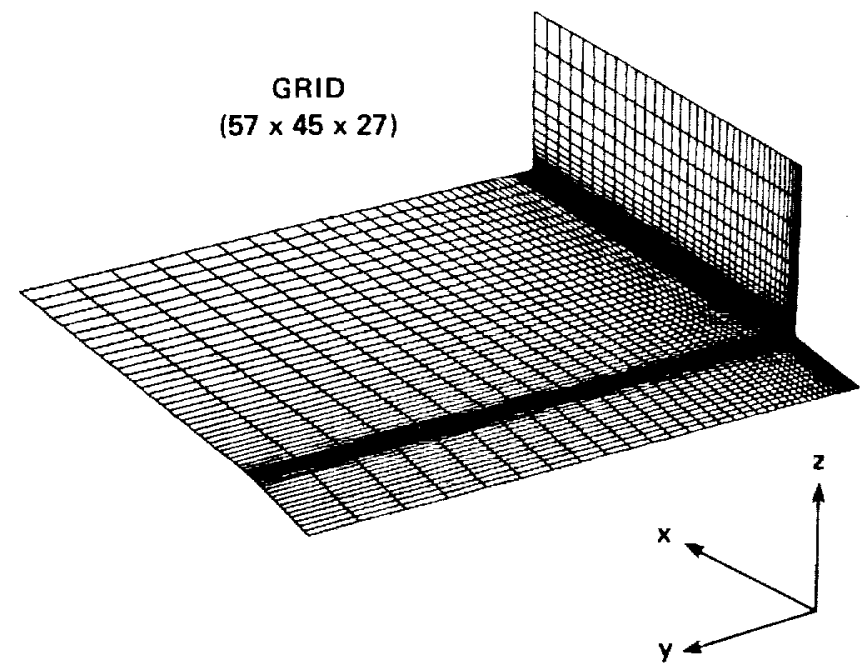

Figure 4.- Mesh system for fin and plate.

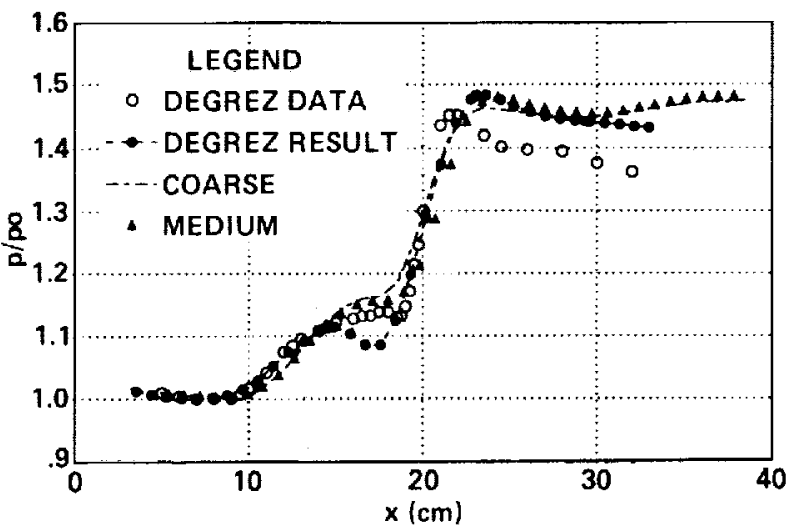

Figure 5.- Comparison of surface pressure at $\mathrm{y}=5 \mathrm{~cm}$.

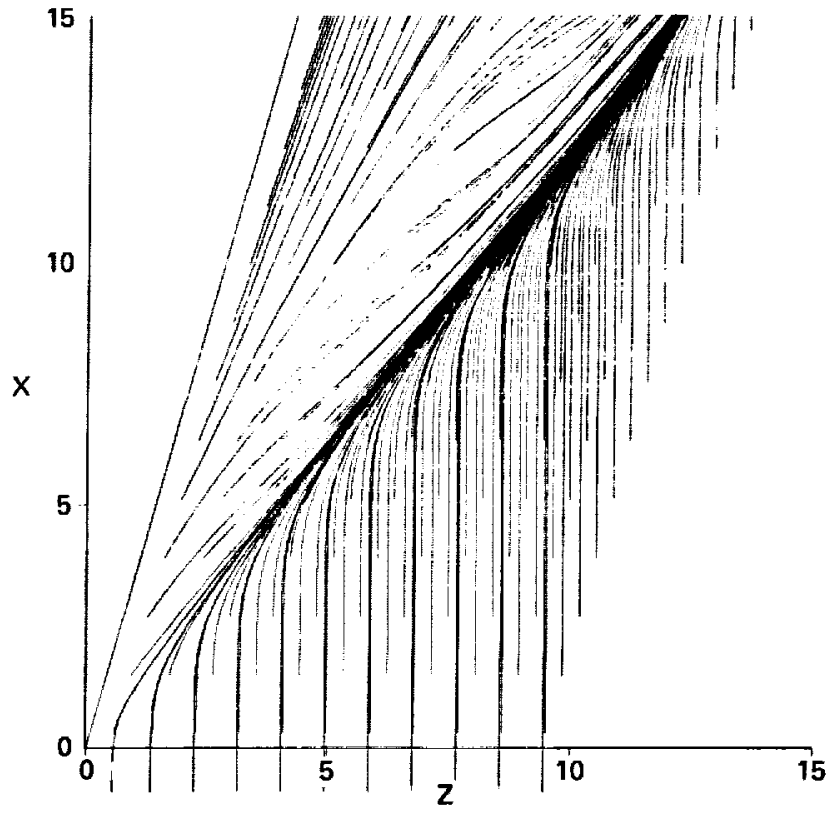

Figure 6.- Surface particle traces by Horstman (ref. 6). 

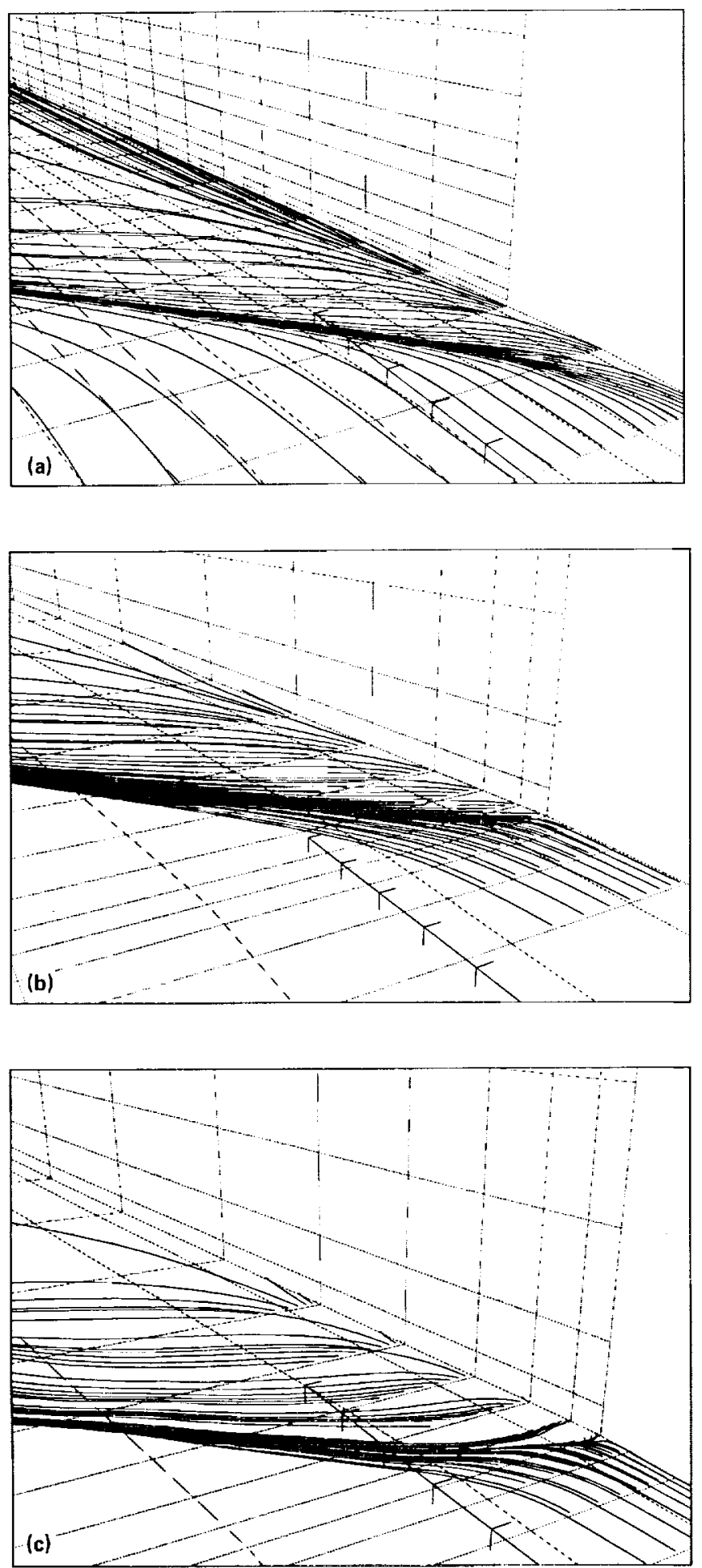

Figure 7.- Surface particle traces $(K=2)$ for $\alpha=6^{\circ}$ : (a) coarse grid; (b) mediun grid; (c) fine grid.

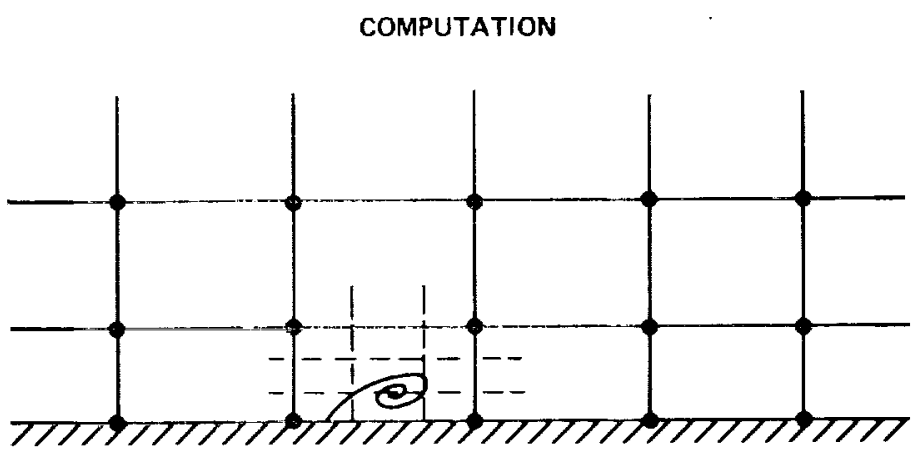

EXPERIMENT

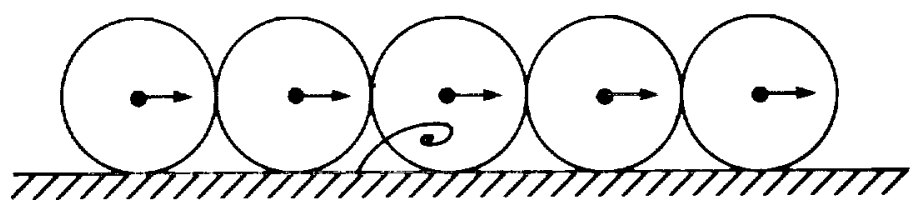

Figure 8.- Resolution problems in computation and experiment.

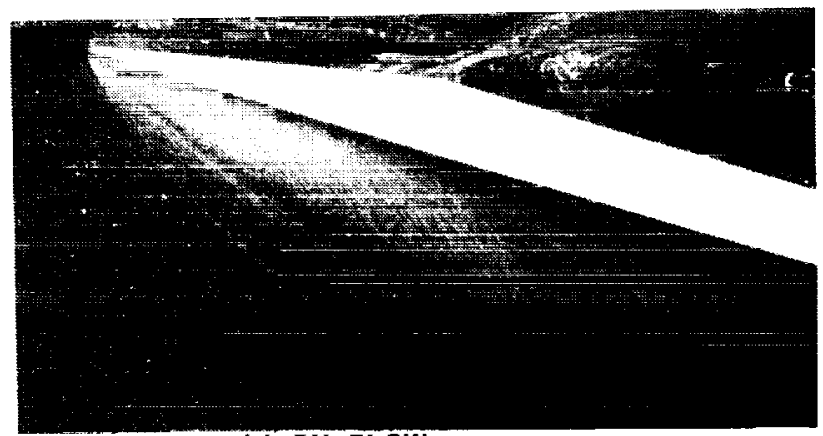

(a) OIL FLOW

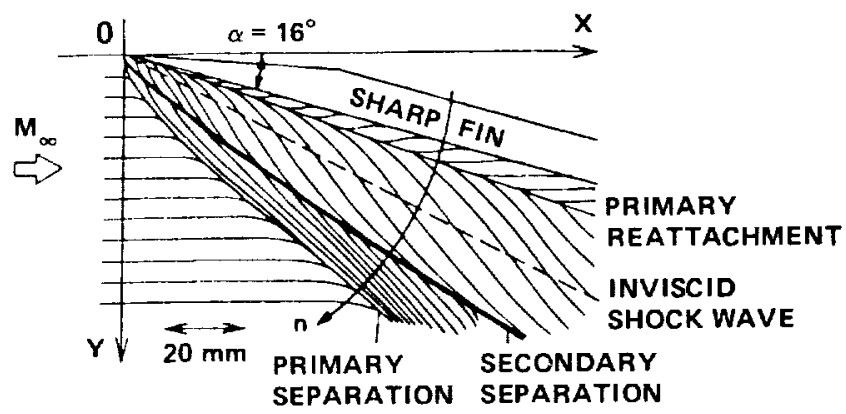

(b) SKETCH OF OIL STREAK LINES

Figure 9.- Surface oil flow by Aso et al. (ref. $13)$. 

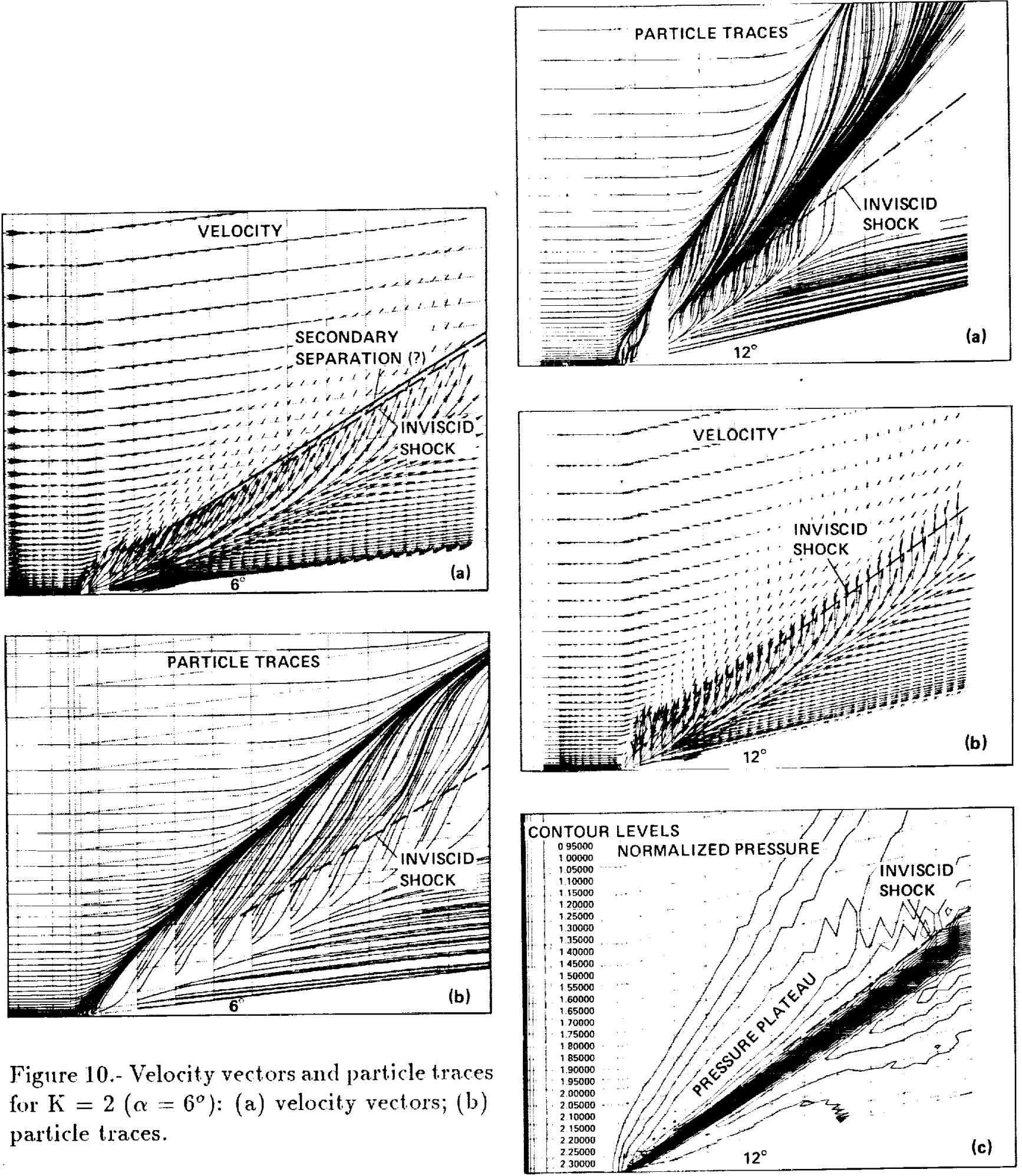

Figure 10.- Velocity vectors and particle traces for $K=2\left(\alpha=6^{\circ}\right)$ : (a) velocity vectors; (b) particle traces.

Figure 11.- Flow properties near the wall (K $=2$ ) for $\alpha=12^{\circ}$ : (a) surface particle traces; (b) velocity vectors; (c) pressure contours. 

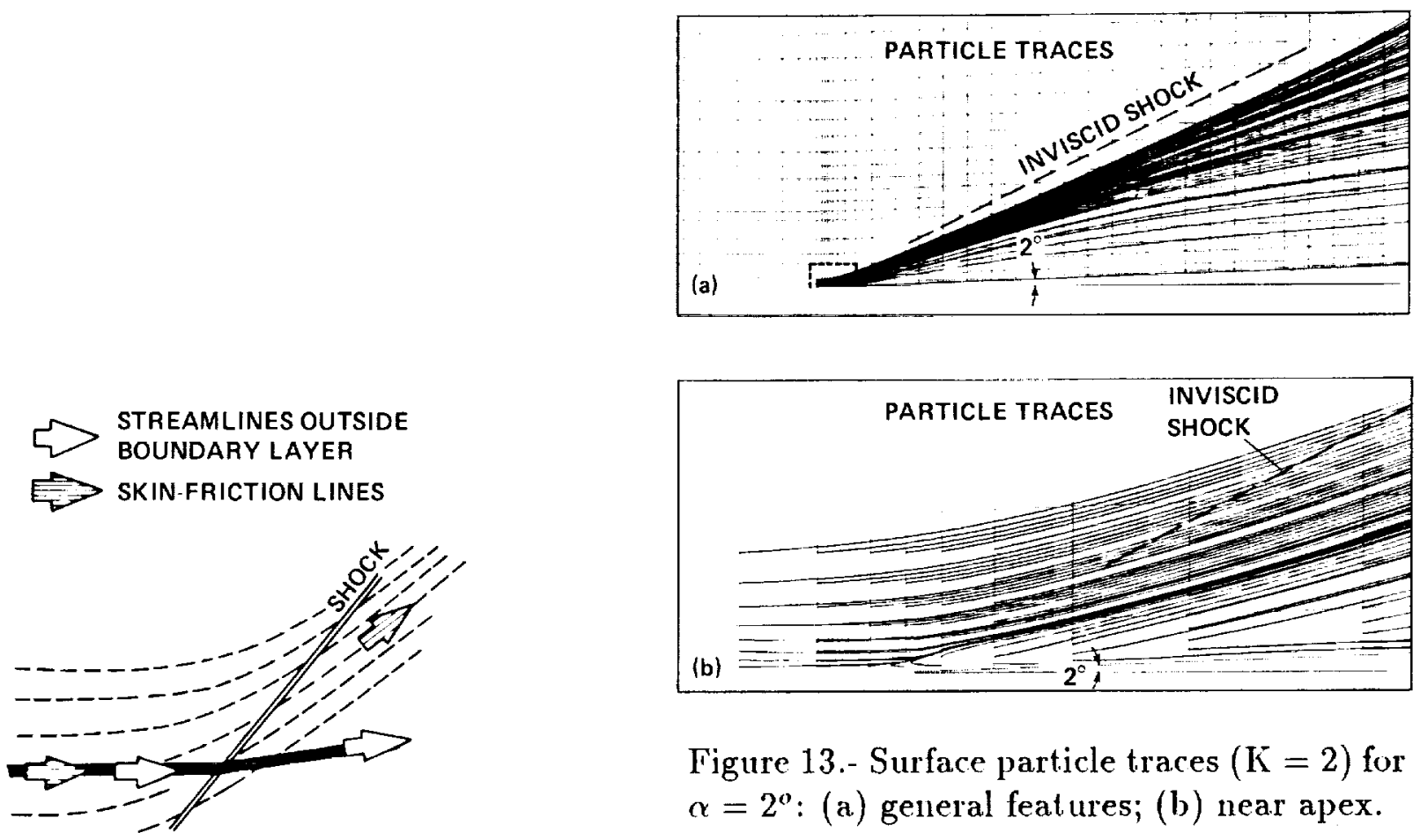

Figure 13.- Surface particle traces $(K=2)$ for $\alpha=2^{\circ}$ : (a) general features; (b) near apex.

(a) ATTACHED FLOW

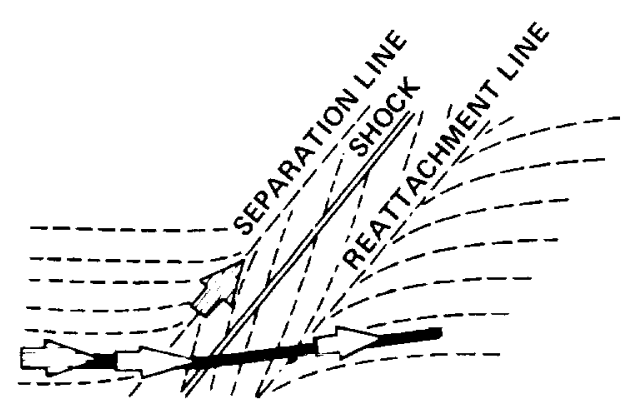

(b) SEPARATED FLOW

Figure 12.- Sketches of skin-friction line patterms beneath glancing shock.

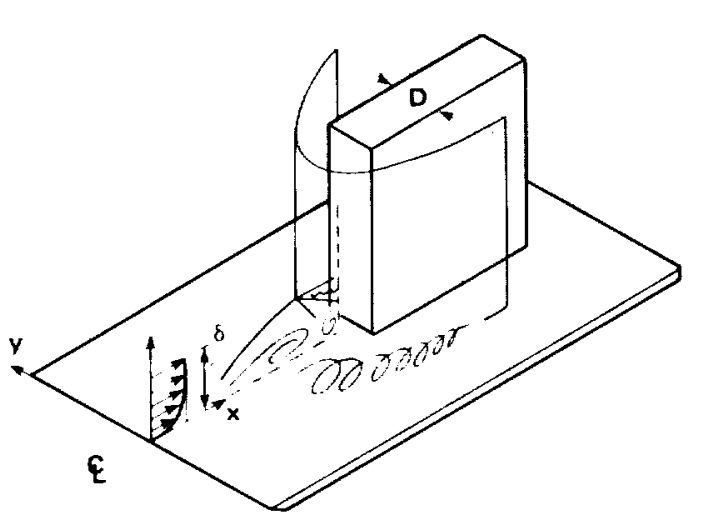

Figure 14.- Supersonic flow over a flat-faced blunt fin on a flat plate. 


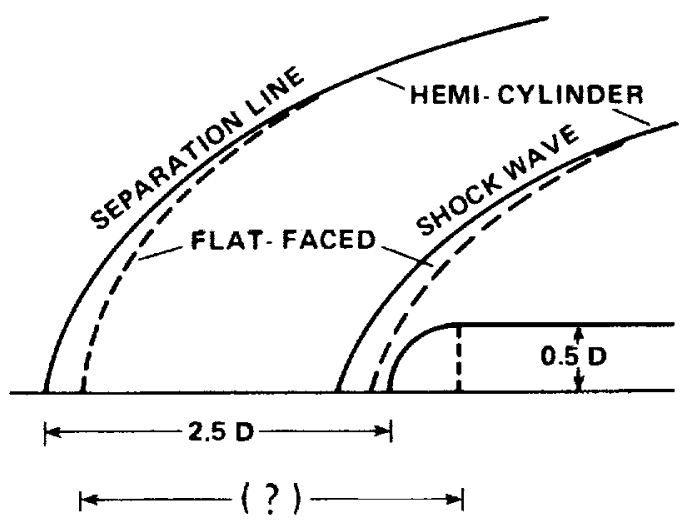

Figure 15.- Sketch of the effects of fin bluntness on separation lines and inviscid shock waves.

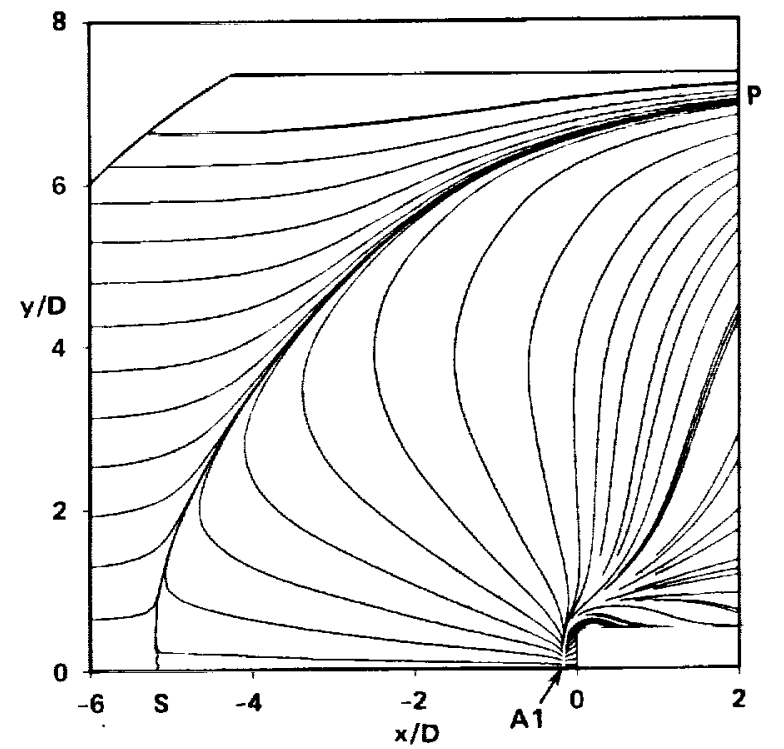

Figure 16.- Surface particle traces $(K=2)$ for blunt fin.

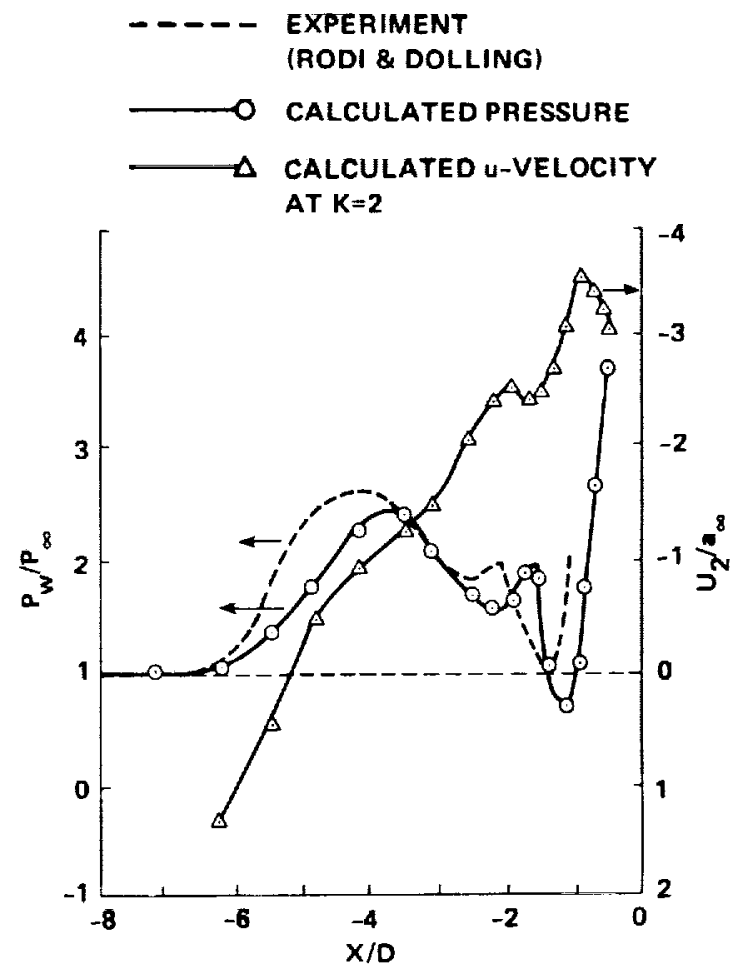

Figure 17.-Comparison of pressure and plot of u-velocity at $\mathrm{K}=2$ along line of symmetry. 


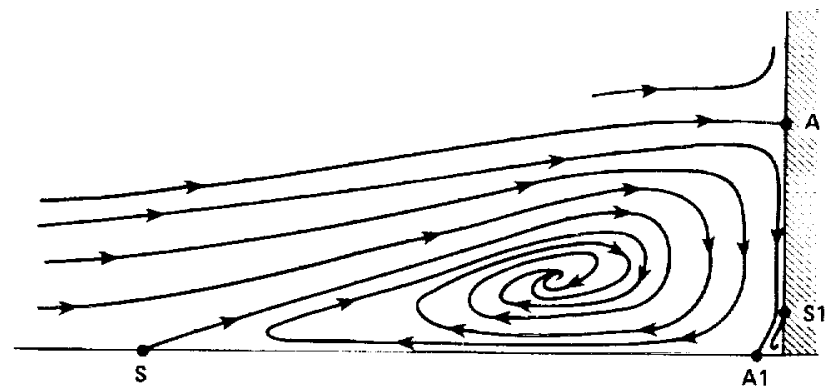

(a) Two Vortices

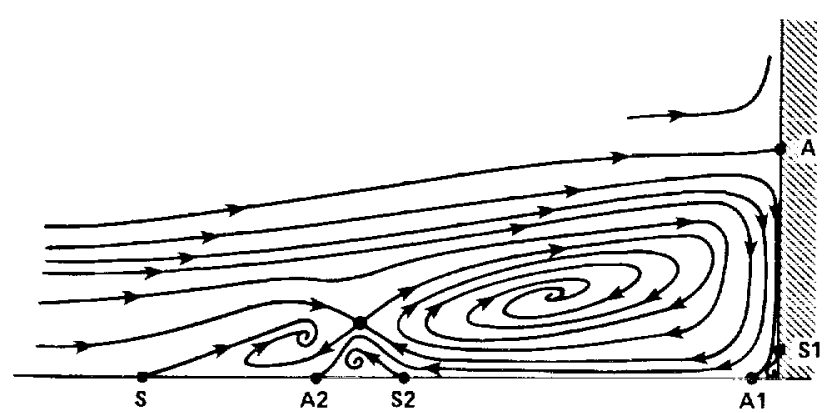

(b) Four Vortices

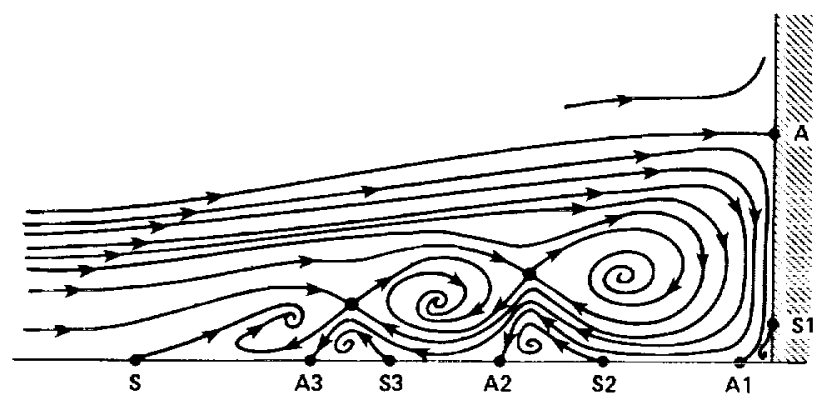

(c) Six Vortices

Figure 18.- Various vortex structures in the plane of symmetry (adapted jet-maze model): (a) two vortices; (b) four vortices; (c) six vortices.

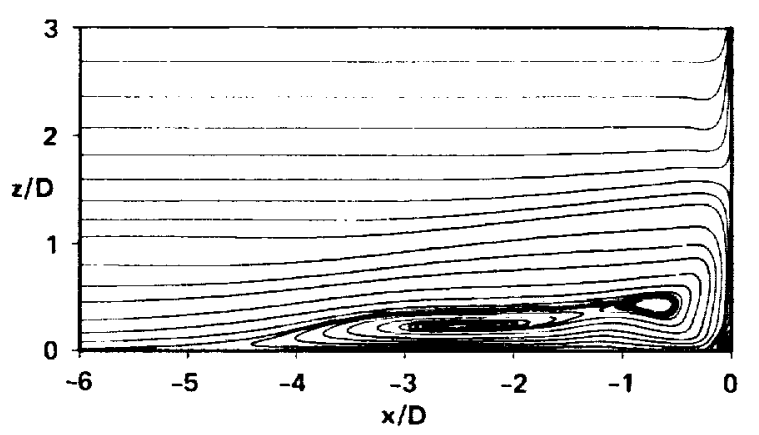

Figure 19.- Particle paths in the plane of symmetry.
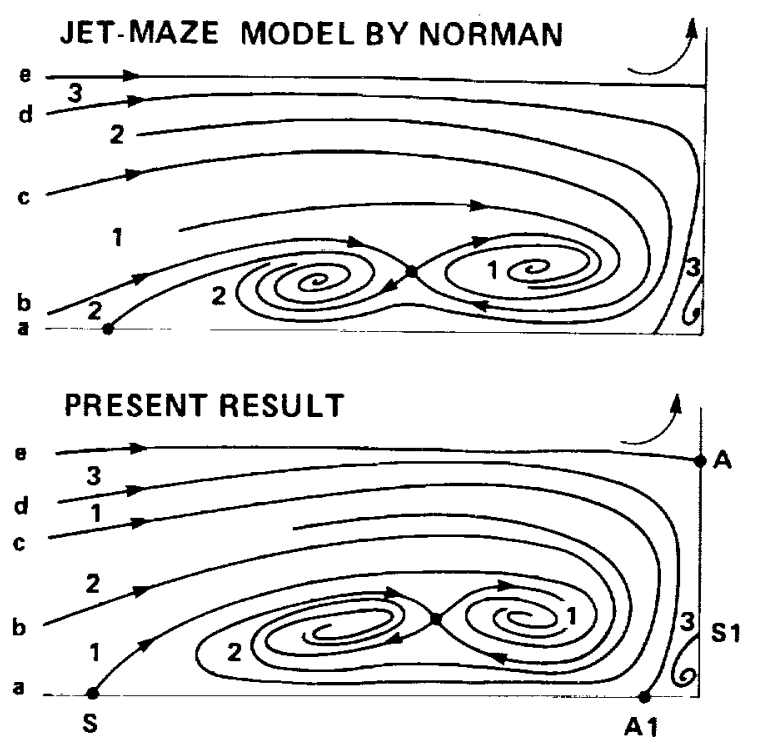

Figure 20.- Structure of horseshoe vortices. 


\begin{tabular}{|c|c|c|c|c|}
\hline 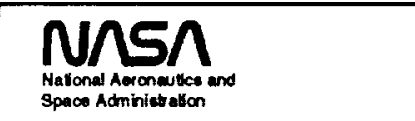 & \multicolumn{4}{|c|}{ Report Documentation Page } \\
\hline $\begin{array}{l}\text { 1. Report No. } \\
\text { NASA TM-102266 }\end{array}$ & \multicolumn{2}{|c|}{ 2. Govermment Accossion No. } & \multicolumn{2}{|c|}{ 3. Recipient's Catalog No. } \\
\hline \multicolumn{2}{|c|}{ 4. Title and Subtitle } & & \multicolumn{2}{|l|}{ 5. Report Date } \\
\hline $\begin{array}{l}\text { Computation of Navier-Stokes Equations for Three-Dimensional } \\
\text { Flow Separation }\end{array}$ & & & \multicolumn{2}{|c|}{ 6. Performing Organization Code } \\
\hline \multirow[t]{2}{*}{$\begin{array}{l}\text { 7. Author(s) } \\
\text { Ching-Mao Hung }\end{array}$} & & & \multicolumn{2}{|c|}{$\begin{array}{l}\text { 8. Performing Organization Report No. } \\
\text { A-90036 }\end{array}$} \\
\hline & & & \multicolumn{2}{|l|}{$\begin{array}{l}\text { 10. Work Unit No. } \\
505-60\end{array}$} \\
\hline \multicolumn{3}{|c|}{$\begin{array}{l}\text { Ames Research Center } \\
\text { Moffett Field, CA 94035-1000 }\end{array}$} & \multicolumn{2}{|c|}{ 11. Contract or Grant No. } \\
\hline \multirow{2}{*}{\multicolumn{3}{|c|}{$\begin{array}{l}\text { 12. Sponsoring Agency Name and Address } \\
\text { National Aeronautics and Space Administration } \\
\text { Washington, DC } 20546-0001\end{array}$}} & \multicolumn{2}{|c|}{$\begin{array}{l}\text { 13. Type of Report and Period Covered } \\
\text { Technical Memorandum }\end{array}$} \\
\hline & & & \multicolumn{2}{|c|}{ 14. Sponsoring Agency Code } \\
\hline \multicolumn{5}{|l|}{ 15. Supplementary Notes } \\
\hline \multicolumn{5}{|l|}{$\begin{aligned} \text { Point of Contact: } & \text { Ching } \\
& (415)\end{aligned}$} \\
\hline \multicolumn{5}{|c|}{$\begin{array}{l}\text { Supersonic flows over a sharp and a flat-faced blunt fin mounted on a flat plate are simulated } \\
\text { numerically. Several basic issues involved in the resultant three-dimensional steady flow separation are } \\
\text { studied. Using the same number of grid points, different grid spacings are employed to investigate the } \\
\text { effects of grid resolution on the origin of the line of separation. Various shock strengths are used to study } \\
\text { the so-called separated and unseparated boundary layer and to establish the existence or absence of } \\
\text { secondary separation. The length of separation ahead of the flat-faced blunt fin, bifurcation of a horseshoe } \\
\text { vortex, and the accessibility of a closed-type separation are investigated. The usual interpretation of the } \\
\text { flow field from previous studies and new interpretations arising from the present simulation are discussed. }\end{array}$} \\
\hline \multicolumn{2}{|c|}{$\begin{array}{l}\text { 17. Key Words (Suggested by Author(s)) } \\
\text { Three-dimensional flow separation } \\
\text { Shock wave/boundary layer interaction } \\
\text { Numerical simulation }\end{array}$} & \multicolumn{3}{|c|}{$\begin{array}{l}\text { 18. Distribution Statement } \\
\text { Unclassified-Unlimited } \\
\text { Subject Category - } 02\end{array}$} \\
\hline $\begin{array}{l}\text { 19. Security Classif. (of this repor } \\
\text { Unclassified }\end{array}$ & $\begin{array}{l}\text { 20. Socuri } \\
\text { Uncl }\end{array}$ & is page) & $\begin{array}{l}\text { 21. No. of Pages } \\
20\end{array}$ & $\begin{array}{r}\text { 22. Price } \\
\mathrm{A} 02\end{array}$ \\
\hline
\end{tabular}

